\title{
Promoting Students' Conceptual Knowledge using Video Analysis on Tablet Computers
}

\author{
Sebastian Becker, ${ }^{1, *}$ Pascal Klein, ${ }^{1}$ and Jochen Kuhn ${ }^{1}$ \\ ${ }^{1}$ University of Kaiserslautern, Dept. of Physics / Physics Education Research Group, \\ Erwin-Schroedinger-Str. 46, 67663 Kaiserslautern, Germany
}

\begin{abstract}
The common idea of video analysis in physics education so far is that students record the motion of an object using a video camera, transfer the video on a computer and analyze it with a suitable software. In our approach students perform video analysis time-efficiently using only tablet computers. We present the results of a study which examines learning effects of tablet PC-supported video analysis in high school physics courses in a pre-post-test design with treatment and control groups for two essential topics of mechanics, the uniform motion ( $N=109$ matched samples) and the accelerated motion $(N=70$ matched samples). The results show that the treatment leads to significantly higher learning gain regarding conceptual knowledge in comparison to traditional teaching without video-based motion analysis, especially for the cognitively more demanding topic. We discuss the results in the context of learning theories and reveal implications for future research.
\end{abstract}

\section{INTRODUCTION}

Digitalization is making a major contribution to our society and will also fundamentally change the teaching and learning culture in schools in particular. On the one hand, this opens up new possibilities for teachers to design teaching-learning processes, but on the other hand it also leads to new requirements which teachers have to face in order to fulfill their educational mission in our contemporary world. It is now the task of education research to support the implementation of digital technologies in regular teaching by gaining empirically secured knowledge about the success factors and benefits of digitally supported teaching and learning scenarios. The present work may contribute to closing the research gap in which the learning impact of classroom scenarios based on the tablet PC-supported video analysis is empirically evaluated.

\section{STATE OF RESEARCH}

The first to investigate the use of smartphones and tablet PCs as an experimental tool in physics were Kuhn and Vogt (2015) [1] in the field of acoustics. A positive learning effect on achievement and motivation could be proven. Based on this, Hochberg et al. (2018) [2] investigated the learning effect of smartphone experiments in the subject of oscillations in secondary-level mechanical education. While no positive learning effect could be diagnosed, effects on curiosity and interest were demonstrated. In this regard, Klein et al. (2018) [3] conceived an application for task-oriented learning with (mobile) video analysis using tablet PCs in the introductory phase of physics and were able to diagnose positive effects on conceptual knowledge and representational competence. Sung et al. (2016) [4] demonstrated in a meta-analysis for the instructional use of mobile digital media a positive medium effect $(g=0.52)$ on the learning performance of the students.

\footnotetext{
* Correspondence should be addressed to s.becker@physik.uni-kl.de
}

For using only tablet PCs, the effect is $g=0.62$. The research findings prove the potential of digital media to effectively support the learning process.

\section{LEARNING THEORY AND HYPOTHESIS}

\section{A. CLT and CTML}

Basic assumption of the Cognitive Load Theory (CLT) (Swellerer (1988) [5], van Merriënboer and Sweller (2005) [6]) is the limited capacity of the working memory. According to the CLT, the primary goal of the design of a learning situation is to keep learning-irrelevant extrinsic cognitive load as low as possible. In the CLT, the split-attention effect is assumed (Sweller and Chandler, (1994) [7]). This (negative) effect implies that the spatial separation of related information sources require mental integration processes, thus increasing extrinsic cognitive load and therefore decreasing the learning-relevant, cognitive load which leads to a reduction in learning performance. The Cognitive Theory of Multimedia Learning (CTML) (Mayer (1999 [8], 2005 [9])) builds on the principle of CLT of a limited capacity of the working memory. In addition, the CTML postulates two separate canals of working memory. A fundamental design principle to prevent the split-attention effect is the principle of contiguity (Mayer and Moreno (2003) [10]). It states that corresponding information should not be presented to the learner in a spatially separate manner and not separated in time. The use of video analysis fulfills this principle by simultaneously displaying real motion sequence and stroboscopic imaging as well as corresponding motion diagrams. The research results of Klein et al. (2018) [3] suggest that video analysis can further reduce extrinsic load by automatically generating and visualizing measurement data. As the cause of this reduction, we assume that by avoiding repetitive, automated activities of learners (such as the acquisition of measurement data) by using technology, the focus on relevant learning objects can be increased and the becoming free cognitive resources are available to the learner for an active knowledge construction. 


\section{B. Hypothesis}

The use of video analysis reduces the extraneous load by automatically generating and visualizing measurement data and fulfills the contiguity principle of the CTML. As a consequence, we expect higher learning gains regarding conceptual knowledge in comparison to traditional teaching methods.

\section{QUASI-EXPERIMENTAL STUDY}

To examine learning effects of tablet PC-supported video analysis and empirically validate the hypothesis, we executed a quasi-experimental study in a pre-post-test design involving high school physics courses which were randomly assigned as treatment group (TG) or control group (CG). The study covered two essential topics of mechanics, the uniform and the accelerated motion. At the beginning of the study, the students had already spent several weeks physics teaching together in the respective courses. The students of the TG used iPad minis and the free application Viana. Before the intervention, the students received a standardized instruction in the handling of the application. A detailed description of the handling can be found in Becker et al. (2016) [11].

\section{A. Samples}

a. Uniform Motion Overall $N=109$ high school students (60\% male) with an average age of 15.7 years $(S D=0.73)$ participated in the entire study. $37 \%$ had physics as a main subject, $21 \%$ mathematics.

b. Accelerated Motion Not all students participated in the follow-up study to the topic accelerated motion. For this reason the sample size was reduced to $N=70$ (57\% male) with an average age of 15.5 years $(S D=0.70)$

\section{B. Study design and material}
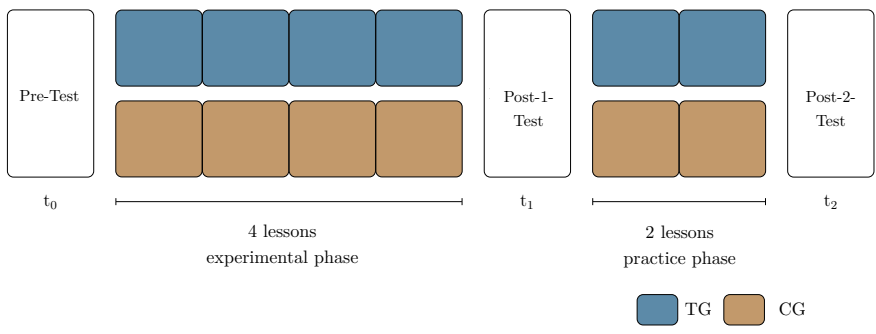

FIG. 1. Study Design: each lesson lasts 45 minutes

The students carried out the teaching unit "Accelerated Motion" immediately after they completed the teaching unit
"Uniform Motion" following an introduction lesson. The design is shown in Fig.1. The prior knowledge of the students was measured directly before the first lesson with a pre-test for the particular topic. The total learning time is divided in an experimental phase and a practice phase. In the experimental phase, the students experimented collaboratively in small groups. In order to allow a fair comparison between TG and $\mathrm{CG}$, the experiments in both groups were identical and the learning tasks were comparable. To ensure this, the development of instructional materials has placed great emphasis on the comparability of materials for both groups in terms of physical learning content, representations used and level of difficulty. Following the experimental phase, the students worked on exercise tasks aimed at consolidating and deepening their knowledge acquired in the experimental phase. The lessons in both phases differed only in the use of technology for the acquisition of measurement data and were otherwise identical for the CG and the TG. In particular, the learning time was exactly the same for both groups. The learning gain for every phase was measured by a post-test containing identical items which allows the empirical determination of the learning gain separately for both phases and the total learning time. Since the interval between the test time points is in the range of one to two weeks, test fatigue or familiarity effects can not be excluded, but should be the same for both groups due to the exact same learning time for TG and $\mathrm{CG}$.

\section{Test instruments}

TABLE I. Sub-concepts of Performance Tests

\begin{tabular}{lll}
\hline \hline $\begin{array}{l}\text { Sub-concept } \\
\text { Uniform Motion }\end{array}$ & Number of items \\
\hline \multicolumn{2}{c}{ G1 } & Velocity as alteration rate \\
G2 & Velocity as vectorial quantity & 6 \\
G3 & Reference system & 5 \\
\hline Accelerated & Motion & 3 \\
\hline B1 & Acceleration as alteration rate & 5 \\
B2 & Acceleration as vectorial quantity & 5 \\
B3 & Reference system & 3 \\
\hline \hline
\end{tabular}

To measure the learning gain, a multiple choice performance test focussing on conceptual knowledge was developed with 18 items for the topic uniform motion and 20 items for the topic accelerated motion. Structuring based on the sub-concepts G1, G2, B1 and B2 was adopted from Lichtenberger et al. (2017) [12] and appropriate items were adapted. The items contain the common forms of representations used in kinematics: diagram, table and strobe. The item pool consists of adapted items from validated test instruments (KCT [12], KiRC [13] and TUG-K [14]) as well as self-developed items. A post hoc item analysis based on Classical Test Theory, Differential Item Functioning and Factor Analysis was 
performed to eliminate unsuitable items from the test instrument before analyzing the students' performance data. The number of items used for performance analysis divided into the basic sub-concepts are listed in Tab. I.

\section{RESULTS AND DISCUSSION}

In order to find significant group differences among the learning gain, the performance data was subjected to an onefactorial analysis of covariance (ANCOVA) regarding the total learning time (period $\left[\mathrm{t}_{0} ; \mathrm{t}_{2}\right]$ ), the experimental phase (period $\left[\mathrm{t}_{0} ; \mathrm{t}_{1}\right]$ ) and the practice phase (period $\left.\left[\mathrm{t}_{1} ; \mathrm{t}_{2}\right]\right)$. The pretest score (or the respective pre-test sub-item score for subconcept analysis) before each learning phase was included as a covariate in the underlying model. The covariate correlates significantly high with the corresponding dependent variable for each learning phase for each topic $(0.51 \leq r \leq 0.85)$. Additionally, the inclusion of the covariate in the model could be justified by a model comparison based on the Akaike information criterion. To compare the learning gain for the sub-concepts between TG and CG, the normalized gain introduced by Hake [15] was determined.

\section{A. Uniform motion}

TABLE II. Group comparison of student test scores for uniform motion; $N=109$ (matched samples); Procedure: ANCOVA with pretest score as covariate.

\begin{tabular}{lccc}
\hline \hline Learning phase & $p$ & $d$ & $1-\beta$ \\
\hline Conceptual knowledge & & & \\
\hline Experimental phase & 0.012 & 0.34 & 0.42 \\
\hline $\begin{array}{l}\text { Sub-concept } G 2 \\
\text { Experimental phase }\end{array}$ & 0.026 & 0.39 & 0.51 \\
\hline $\begin{array}{l}\text { Sub-concept G3 } \\
\text { Experimental phase }\end{array}$ & $<10^{-3}$ & 0.64 & 0.91 \\
\hline \hline
\end{tabular}

Results of ANCOVA are shown in Table II. For the experimental phase, a significant group difference can be determined in favor of the TG with a small effect size and only poor test power $(d=0.34,1-\beta=0.42)$. The enhanced learning gain of the TG can be reduced to sub-concepts $\mathrm{G} 2$ and $\mathrm{G} 3$. In particular, a highly significant group difference in learning gain with respect to the sub-concept G3 with a medium effect size and high test power $(d=0.64,1-\beta=0.91)$ could be detected. However, the deficits of the $\mathrm{CG}$ in the experimental phase could be compensated during the practice phase.
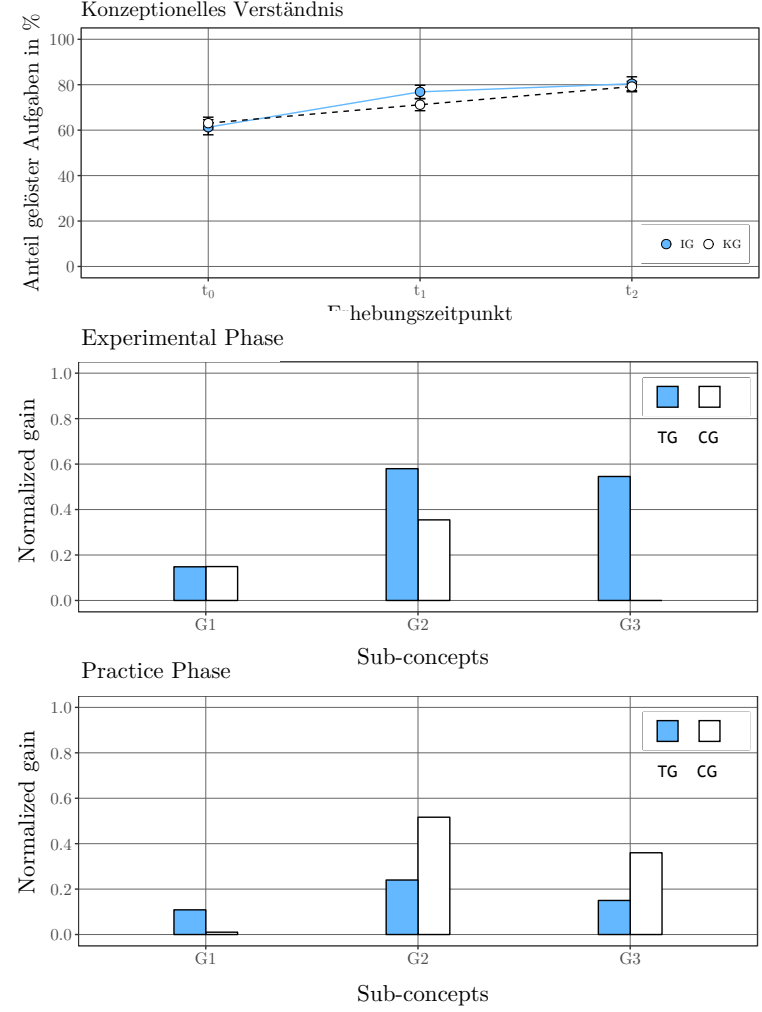

FIG. 2. Results for the topic uniform motion: group mean and standard error (top panel), normalized learning gain in experimental and practice phase (bottom panel)

TABLE III. Group comparison of student test scores for accelerated motion; $N=70$ (matched samples); Procedure: ANCOVA with pretest score as covariate.

\begin{tabular}{lccc}
\hline \hline $\begin{array}{l}\text { Learning phase } \\
\text { Conceptual knowledge }\end{array}$ & $p$ & $d$ & $1-\beta$ \\
\hline Experimental phase & 0.002 & 0.63 & 0.73 \\
Practice Phase & 0.006 & 0.43 & 0.42 \\
Total learning time & $<10^{-3}$ & 0.92 & 0.96 \\
\hline Sub-concept B1 & & & \\
Experimental phase & 0.022 & 0.46 & 0.46 \\
Practice Phase & 0.018 & 0.33 & 0.27 \\
Total learning time & 0.001 & 0.67 & 0.78 \\
\hline Sub-concept B2 & & & \\
Experimental phase & 0.001 & 0.80 & 0.90 \\
Total learning time & 0.001 & 0.71 & 0.83 \\
\hline
\end{tabular}

\section{B. Accelerated motion}

Results of ANCOVA are shown in Table III. Regarding the complete learning time, a large effect for the benefit of the TG could be verified with high test power $(d=0.92$, $1-\beta=0.96$ ). If we compare the learning performance for 


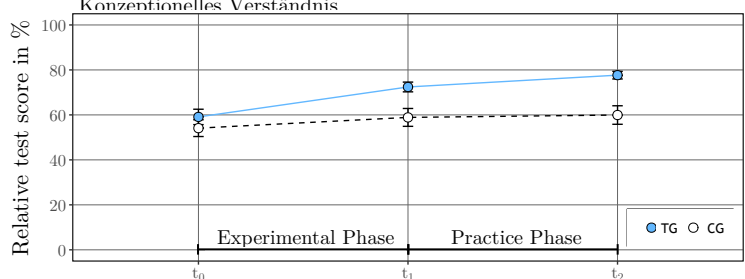

Point in time

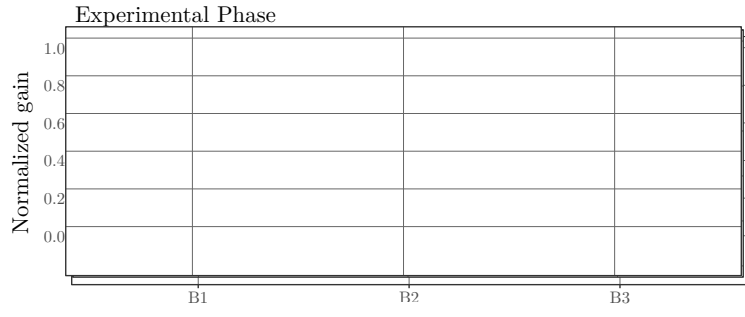

Sub-concepts

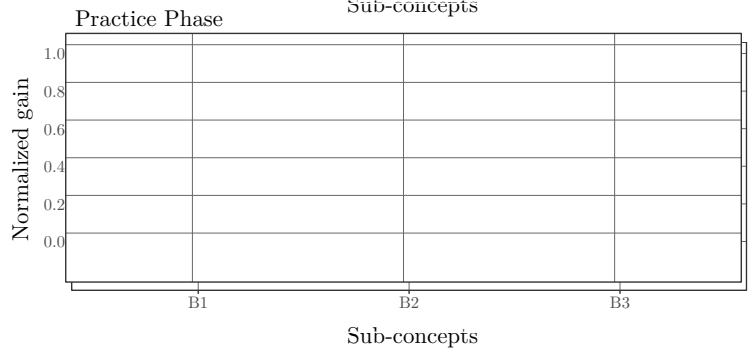

FIG. 3. Results for the topic accelerated motion: group mean and standard error (on top), normalized learning gain in experimental and practice phase (below)

both experimental and practice phase, the effect is larger for the experimental phase (medium effect, $d=0.63$ ) than the practice phase (small effect, $d=0.43$ ). This coincides with the results for the topic uniform motion, which indicates a stronger effect in the experimental phase. The higher learning gain of the students from the TG can be attributed to sub-concepts B1 and B2 and not B3. Medium to large effects for the sub-concept B2 could be statistically validated with high test power, both for the experimental phase (large effect, $d=0.80,1-\beta=0.90$ ) and the total learning time (medium effect, $d=0.71,1-\beta=0.83$ ). With regard to the entire learning time, a medium effect in favor of the TG with high test power is also detectable for the sub-concept B1 $(d=$ $0.67,1-\beta=0.78$ ). In summary, the validity of the hypothesis can be empirically supported for both experimental phase and the total learning time with high test power, for the practice phase with poor test power. Comparing the learning performance for both topics, a clearly larger effect could be proven for the cognitively more demanding topic.

\section{CONCLUSIONS AND OUTLOOK}

The quasi-experimental study presented in this work was aimed at the empirical investigation of the learning effectiveness of tablet PC-supported video analysis. For this purpose, the digital learning tool has been used by teachers in regular teaching in the topics of uniform motion and accelerated motion. By comparing the learning performance with a control group, which was taught traditionally, the assumed higher learning effectiveness of the tablet PC-supported video analysis could be empirically confirmed in the conceptional understanding. In the experimental phase, the learners benefited more from the use of video analysis than during the practice phase. It should be noted that the learning-related effect, based on the assessment used, was demonstrated for a special teaching scenario covering specific topics of mechanics with a manageable sample size. However, the research results suggest not only that an implementation of a digital learning tool into regular teaching can succeed, but also that a digitallysupported lesson can be superior to traditional teaching with regard to the learning performance of the students. In order to generalize the research results of this work and to empirically investigate motivational variables and their impact on learning performance, a reproductive study with a larger sample size has already been carried out.
[1] J. Kuhn and P. Vogt, Multidisciplinary Research on Teaching and Learning, , 253 (2015).

[2] K. Hochberg, J. Kuhn, and A. Müller, Journal of Science Education and Technology 27, 385 (2018).

[3] P. Klein, J. Kuhn, and A. Müller, Zeitschrift für Didaktik der Naturwissenschaften , 1 (2018).

[4] Y.-T. Sung, K.-E. Chang, and T.-C. Liu, Computers \& Education 94, 252 (2016).

[5] J. Sweller, Cognitive Science 12, 257 (1988).

[6] J. J. G. van Merriënboer and J. Sweller, Educational Psychology Review 17, 147 (2005).

[7] J. Sweller and P. Chandler, Cogntition and Instruction 12, 185 (1994).

[8] R. Mayer, International Journal of Educational Research 31, 611 (1999).
[9] R. Mayer, Cognitive theory of multimedia learning, Vol. 31 (Cambridge University Press, 2005) pp. 31-48.

[10] R. Mayer and R. Moreno, Educational psychologist 38, 43 (2003).

[11] S. Becker, P. Klein, and J. Kuhn, The Physics Teacher 54, 440 (2016).

[12] A. Lichtenberger, C. Wagner, S. I. Hofer, E. Stern, and A. Vaterlaus, Physical Review Physics Education Research 13, 010115 (2017).

[13] P. Klein, A. Müller, and J. Kuhn, Physical Review Physics Education Research 13, 010132 (2017).

[14] R. Beichner, American Journal of Physics 62, 750 (1994).

[15] R. R. Hake, American Journal of Physics 66, 64 (1998). 